\title{
COMPARING UCN WITH FDDI AND DQDB USING A REALISTIC WORKLOAD MODEL
}

\author{
H.L. Pasch and I.G. Niemegeers \\ Tele-Informatics and Open Systems Group \\ University of Twente \\ P.O. Box 217, 7500 AE Enschede, The Netherlands
}

\begin{abstract}
The token ring FDDI and the slotted bus $D Q D B$ are the two currently most important standards for high speed networks. Each of these networks has its own specific advantages and disadvantages, and its own specific type of load for which it performs best. We propose a new network design, called Universal Channel Network (UCN). UCN is adaptive and can be made to behave either like a token ring, a slotted ring, or anything in between. The aim is to get a network that combines the advantages of both the token ring and the slotted ring. In this paper $U C N$ is explained and its performance is compared with that of DQDB and FDDI. The workload model used is a detailed representation of today's traffic, extrapolated into the future.
\end{abstract}

\section{Introduction}

Over the last 20 years a lot of research has been conducted on new access mechanisms for LANs, and more recently for High Speed LANs and MANs. One of the main concerns has been the performance, in particular the transfer delay. Most of these networks are designed to perform at their best for a particular type of load (usually data traffic), but their performance decreases under loads that have strongly different characteristics. Because of the uncertainty about the characteristics and the mix of future (broadband) traffic (see e.g. [1] and [2]) it is important to search for networks which can adapt to the traffic characteristics.

Two access mechanisms that are to a certain extent complementary when it comes to the influence of the message length on the performance, are the token ring and the slotted ring. It has been shown in [3] and [4] that for various types of slotted and token rings, the latter perform significantly better for long messages, while the former are at their best for short messages. Ideally a network should perform well for both types of load, i.e. it should combine the advantages of both slotted and token rings in a single network. In this paper we propose a design which goes in this direction, and compare it against FDDI (-II) and DQDB.

In Section 2 of this paper we analyse the difference between the slotted and the token ring, focusing on the performance. In Section 3, we introduce the basic concepts of a new network called Universal Channel Network (UCN), which is aimed at HSLANs and MANs. We argue that UCN combines the advantages of the slotted and the token ring, by making the mechanism that causes the difference in performance adaptive. In Section 4 we discuss the workload model used to derive the simulation results presented in Section 5. In Section 6 we draw some conclusions and list some ongoing work concerning UCN.

\section{Comparing slotted rings and token rings}

In this section the difference between slotted rings and token rings will be examined. It will be shown in both a qualitative and a quantitative way what the difference is between them, how this influences performance, and how token ring and slotted ring can be combined.

In the sequel we will repeatedly use the term message with the meaning of a Data Link Layer Service Data Unit (DLL SDU).

\subsection{Qualitative considerations}

The station that holds the token in a token ring has the exclusive right to use the ring. Similarly in a slotted ring, the station which changed the busy flag of a slot from 'idle' to 'busy' has the exclusive right to use the particular slot. This right is relinquished by putting the (free) token back onto the ring or by changing the busy flag from 'busy' back to 'idle', respectively. Therefore in case of the slotted ring we will also speak of the token of a slot when we mean the exclusive right to use the slot.

The fundamental difference between the two systems is that in the token ring there is a single token that controls the access to the entire bandwidth, whereas in the slotted ring, there are a number of tokens, each controlling access to part of the bandwidth (the transfer capacity of a single slot recurring periodically).

This difference is also reflected in the performance models of the two systems: they can be modelled by single and multiple cyclic server queueing models respectively [7]. Let us now explain how this difference also causes the difference in performance characteristics between the token ring and the slotted ring 1 .

\subsection{Performance considerations}

In [3] an extensive performance evaluation has been made of a number of high-speed ring protocols. Among the protocols

1. There is also another major difference between a token ring and a slotted ring. We discuss this difference later on. 
that have been compared are the multiple token ring with exhaustive service and a slotted ring with a combination of normal and channel slots and in which a station can use several slots simultaneously. The influence of the average message length on the mean total sojoum time ${ }^{2}$ that a message suffers has been investigated. The performance evaluation shows that the token ring outperforms the slotted ring when the average message length is large. For a short average message length, however, the slotted ring outperforms the token ring. In other words: for a given message length the performance depends, amongst other parameters, on the number of tokens. Therefore, for each type of load there is a particular number of tokens that provides the best performance.

This property can be explained by efficiency arguments. For long messages the token-passing mechanism becomes more and more efficient, since the ratio of the overhead per message and the data field (SDU) is small and decreases as the message length increases. For slotted rings this is not the case. Long messages are segmented into mini-packets, which fit in a slot, and which have a constant amount of overhead. As the message length increases, the overhead grows quasi linearly. The net result is that for a given effective network utilization (MAC SDU traffic load), the gross utilization of the transmission medium will be larger for slotted rings than for token rings, leading to lower expected sojourn time for the latter.

For short messages on the other hand the opposite occurs. Here two effects cause the token passing mechanism to become less and less efficient as the average message size decreases. First there is the token passing overhead due to preamble (assuming an asynchronous transmission scheme) and $\mathrm{PCI}$, which is larger than the overhead per mini-packet in a slotted ring. Typical values are 200 bits for token passing vs. 48 bits for a high-speed slotted ring [7]. The second effect occurs at low and medium loads. When the message transmission time becomes short compared to the ring latency, the token latency, i.e. the time a message at the head of a station queue has to wait until a free token arrives, starts playing an important role. A token ring, with its single free token is here at a disadvantage, compared to a slotted ring with multiple slots, where a number of free tokens are circulating. This becomes particularly important when, for a given type of traffic load, transmission speed is increased.

The conclusion one can draw is that for long messages the token-passing mechanism is more efficient and tends to offer better delay performance, while for short messages the slotted ring is better. An important design goal of UCN is that its behaviour can be varied in discrete steps between that of a ring with multiple slots and that of an efficient token passing ring. In UCN the number of slots, i.e. the number of

2. With sojoum time we mean the time interval from the arrival of the first bit of a message at a (source) station until the last bit of the message has been received by the destination. tokens can be adapted to the traffic characteristics. In particular for a given average message size the number of slots or the slot size can be optimized with respect to the expected sojourn time. In [8] it is shown that such an optimum exists.

\section{The UCN access mechanism}

In this section we discuss specific features of the UCN ring: the slot layout (Section 3.1), and the grouping mechanism which adapts the slot size by joining adjacent basic slots (Section 3.2). We further argue in Section 3.3 that the UCN ring can be made to behave like a multiple token ring, with the corresponding efficiency. In Section 4 we present some simulation results that show the gain that is achieved by adapting the group sizes to the load.

\subsection{Slot layout}

$\mathrm{UCN}$ is a slotted ring (see Figure 1). Every slot (also referred to in this paper as a basic slot) consists of:

- a Token bit (T-bit): Access to a slot is controlled by the token bit. A free slot is characterised by a T-bit equal to 1. A station captures a free slot by setting the $T$-bit equal to 0 . It can use the slot as long as it needs it (within the constraints of the gated service discipline). A slot is released by changing the T-bit back to 1 . Several slots can be used simultaneously by a single station.

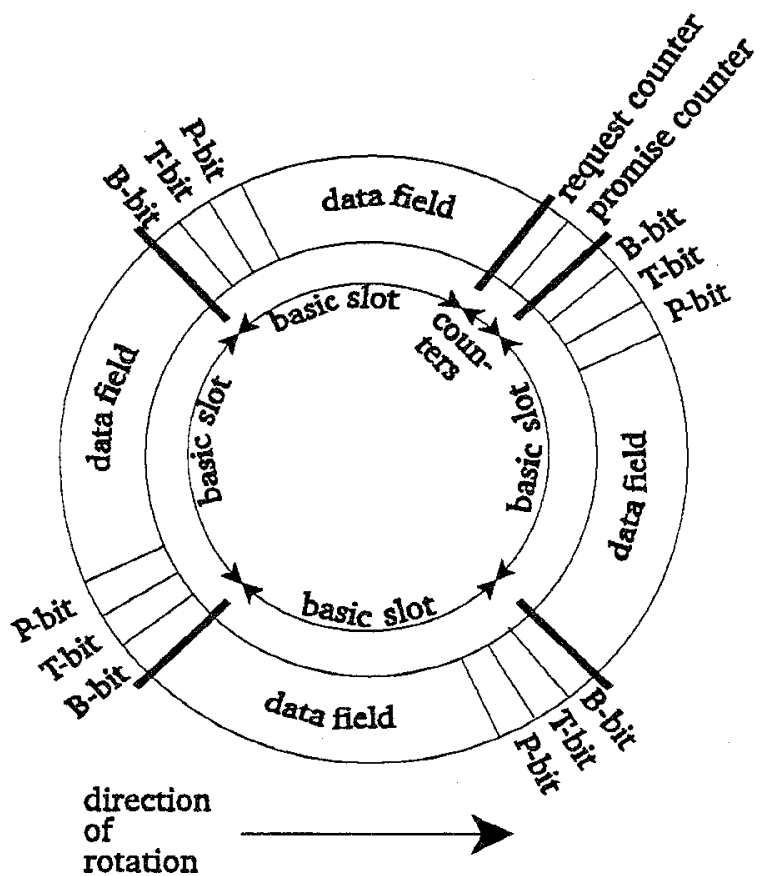

Figure 1: Layout of the UCN ring.

a Contain_PCI bit (P-bit): In UCN a station can use a slot repeatedly for transferring successive parts of a message. 
Only the first time a slot is used it has to contain the PCI since afterwards both sender and receiver know that this particular slot is used for transferring a certain message (Figure 2). The P-bit indicates that a slot contains the beginning of a message. This allows substantial savings in overhead compared to the slotted-ring mechanism used e.g. in the Cambridge Fast Ring [6] and Cambridge Backbone Network [9].

- a Beginning_of_group bit (B-bit): In the initial situation every basic slot is separately accessible and therefore has its own token. Adjacent basic slots can be joined to form a group ${ }^{3}$, i.e. a single large slot with a single token. A group can consists of any number of basic slots, ranging from one to all the basic slots on the entire UCN ring. Basic slots keep their B-, T- and P-bits when they are joined. The B_bit is used for marking the border between adjacent groups. A B-bit set to 1 indicates the beginning of a new group, while a B-bit set to 0 indicates that a basic slot belongs to the same group as the previous one.

- a data field. This field contains segments of the message (Figure 2).

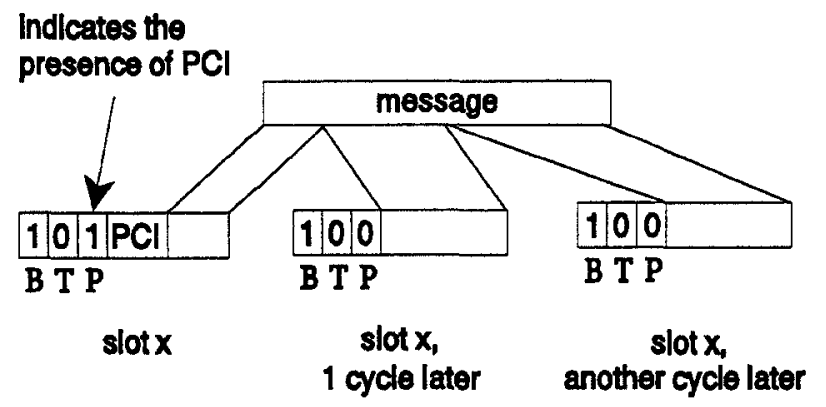

Figure 2: Segmenting messages

Except for slots, the UCN ring also contains two counters (the promise counter and the request counter), on which the UCN priority mechanism is based. See [13] for details on the priority mechanism.

\subsection{Grouping}

As we saw, the way a system performs depends on the number of tokens, and therefore on the size of a group. Let us describe how a station can change the group size. The initial situation is illustrated in Figure 3a: two groups consisting of a single basic slot each. Group 1 and 2 are joined by changing the first (and in this case the only) B-bit of group 2 from 1 to 0 and by removing the token from group 2, because access to a group is controlled by a single token. The resulting situation is illustrated in Figure $3 \mathrm{~b}$.

The size of a group can be decreased by splitting a group into a number of smaller groups, each consisting of an

3. The term 'group' is introduced (instead of using the term 'slot') to prevent confusion with the term 'basic slot'. integer number of basic slots. It is necessary to mark the beginning of each new group with a B-bit of 1 , and to put a token into each of the new groups. This process of splitting is exactly the opposite of grouping.

Adapting the group size to the general characteristics of the traffic is only done by a management station. Grouping could be based on monitoring the traffic on the ring or on apriori knowledge about the network load as a function of the time of the day, e.g. mainly short-message telephone traffic between 9.30 and $11.00 \mathrm{am}$, and large file transfers between 4 and $5 \mathrm{pm}$.

\subsection{Early token release}

We have explained how the number of slots and hence also the slot size can be adapted in the UCN ring. By decreasing the number of slots to one we claim that the system can be made to approach the behaviour of a multiple token ring quite closely, provided the size of the basic slots is sufficiently small compared to the average message length. Let us give the qualitative arguments which lead to this conclusion.

In both a slotted ring containing a single slot, and a multipletoken ring, access to the entire bandwidth is controlled by a single token. However there are still significant differences:

- If, in a multiple-token ring, a station has a backlog of several messages, the transmission of the next message is started immediately after the transmission of the previous message. Furthermore the token is released immediately after the transmission of the last message. Waste of transmission capacity is limited to the passing of the token from station to station, i.e. it is determined by the ring latency.

- In a slotted ring a message has to start at the beginning of a slot. Therefore when a message does not exactly fit in an integer number of slots, waste occurs due to internal fragmentation. Furthermore, since a token is passed to a station at the beginning of a slot, the time between the end of the last message a station has to transmit and the beginning of the next slot is wasted. This type of waste generally increases with the slot size for a given message-length distribution. It can therefore be substantial in case of a ring containing a single, large slot.

Hence, compared to the multiple-token ring, the slotted ring has some inefficiencies which tend to become worse when the slot size increases. In UCN this problem has been significantly reduced because large slots, the groups, have a substructure of basic slots. A message can in principle start at the beginning of each basic slot within a group, and not just at the beginning of a group. So, if a message ends and leaves a number of basic slots within a group unused, they are not wasted, but used for the transfer of the next message. 


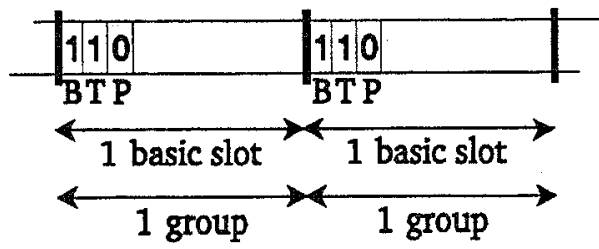

Figure 3a: grouping

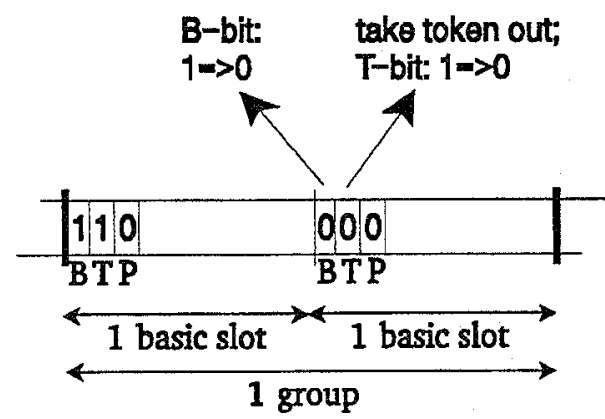

Figure $3 b$

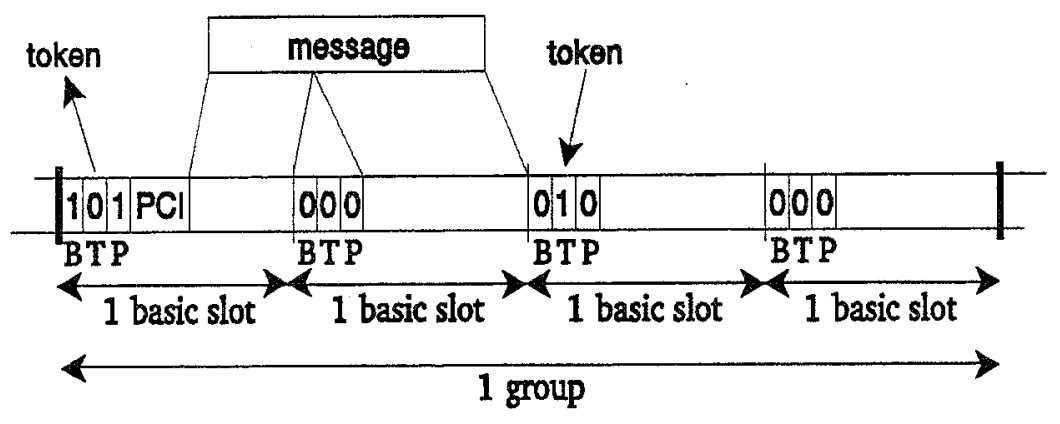

Figure 4a: Early token release

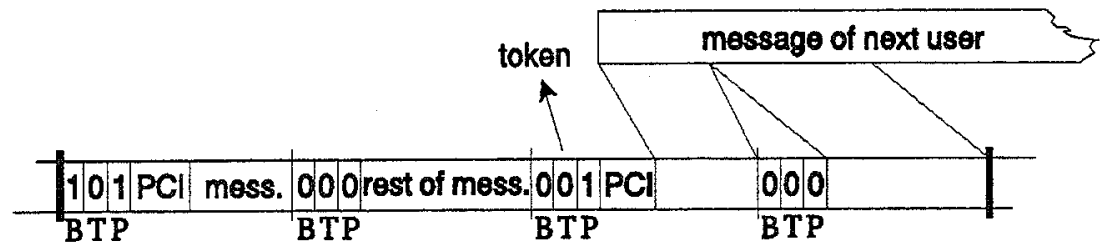

Figure $4 b$
Fragmentation only occurs within a basic slot and does not depend on the group size.

The same holds for the passing of the token. While in ordinary slotted rings the token is put at the beginning of a slot, in UCN it can be put at the beginning of each basic slot of a group, therefore minimizing the waste. An example will clarify this. Consider a group consisting of four basic slots (Figure 4a) and a message with a length of two basic slots. Assume further that in our initial situation the token is in the first basic slot of the group. The station claims the token, changes the P-bit of the first basic slot to 1 and puts its message into the slot. After the message has been transmitted, the token of the group is released by putting it into the next basic slot (basic slot number 3) of the group. The next backlogged station will take the token out of this basic slot, change the P-bit to 1 and start transmitting its message (Figure 4b).

We saw that in UCN the amount of waste depends on the basic slot size, and not on the group size. We now discuss why the size of a basic slot can be chosen very small in order to minimize waste and the difference between the behaviour of UCN and a multiple token ring with exhaustive service.

In most slotted systems the slot size is a trade-off between two types of waste:

- PCI waste: since normally every slot needs PCI, an increase in the slot size means that a message can be transferred using less slots and therefore with less overhead;

- Fragmentation waste: a message does not normally fill up an integer number of slots; on the average the last slot will only be filled half; therefore, the larger the slots the larger the fragmentation waste.

In UCN, however, PCI is not sent once per basic slot, but once per group, regardless of how often the group is used. Therefore the PCI overhead is independent of the basic slot size which can be decreased, without incurring an efficiency penalty, in order to minimize the fragmentation waste. This further minimizes the difference between the behaviour of $\mathrm{UCN}$ and the multiple token ring with exhaustive service.

\section{Workload model}

In [13] the performance of UCN is analysed and compared with that of a token ring and a slotted ring. A simple workload model has been used that makes the differences between the networks very clear. It is shown that UCN outperforms the (multiple) token ring and the (channel) slotted ring for a broad spectrum of loads. This is caused by the fact that the performance of UCN can be adapted to the situation under which it has to operate. In [20] it is further shown that an exact adjustment is not crucial for a good performance of UCN.

In this paper UCN is compared with the DQDB [24] and FDDI-II ([22],[23]) using a realistic workload model. This section describes the workload model; the next section describes the simulation results achieved.

\subsection{Introduction}

Based on network traffic measurements ([16],[17]) and knowledge of the operation of current higher layer protocols, a detailed workload model has been developed. This 
workload model represents network traffic in a scientific/campus environment. Next, this workload model has been extrapolated into the future, resulting in a model for the workload as it may be seen in future. Detailed information on the workload model can be found in [21].

In order to get realistic arrival processes, a so-called requestreply chain is used in modelling. We start with explaining what we mean by that. Next we list the applications that are modelled. Then the modelling of each of these applications is described.

It is not our intention to present 'the' workload model of future integrated services traffic, but to present 'a' possible workload model. By making a quite realistic and detailed workload model we intend to include all the elements that are relevant to the performance, and that are not included in most simple workload models.

\subsection{The request-reply chain}

Poisson processes are commonly used as the arrival process in analytical studies and in simulations. However, Poisson processes are not able to model some special behaviour of message arrivals, e.g. bursty arrivals and bulk data transfer. In addition, measurements of traffic on local area networks showed that the arrivals of successive messages have a correlation with one another which strongly influences the overall system performance ([15], [16], [17], [18], [19]). The correlation between arrivals stems mainly from acknowledgements, retransmissions, and replies in requestreply protocols. Therefore we use the request-reply chain in some workload components: the receipt of a message at a station triggers the generation of the next message. The processing time of a message is incorporated by letting some time expire between the receipt of a message and the generation of its reply.

\subsection{Applications}

Each network is modelled to consist of three different types of nodes:

- 1 server node, with the file servers and paging servers connected to it;

- 1 gateway node, through which all the communication with the outside world occurs;

- the rest of the nodes are workstation nodes, with one or more workstations, telephones, and videophones attached to it.

Based on these node types the following applications are distinguished:

- real-time audio and video telephony;

- file access;

- swapping;

- character traffic.

Each of these components will now be addressed in short.

\subsection{Telephony and videophony}

Telephones and videophones generate isochronous data streams with intensities that are multiples of $64 \mathrm{kbit} / \mathrm{s}$. These streams are mapped onto isochronous channels ${ }^{4}$. All three networks offer isochronous channels.

\subsection{File access}

When a workstation has to execute a command or program, the code of this command or program is downloaded from the file server to the workstation. This is called file access. File access also includes the transfer of data files from file server to workstation and the other way around (e.g. a text file in case of text editing).

Files are accessed in quantities of 1 page at a time. A file page is an operating system quantity. File access has been modelled as a request-reply chain. After a chain has been initiated by a workstation, several short messages are exchanged between workstation and file server. This eventually results in the transmission of a file page either from server to workstation, or from workstation to server.

In many current systems the next file page can only be requested after the previous one has completely been received. This may become a throughput bottle-neck in future. In our extrapolated workload model this limitation has been removed. In the extrapolated workload model workstations initiate file page requests hyper-exponentially.

\subsection{Swapping}

In case a workstation application runs short of physical memory (RAM), it tries to get rid of RAM content that has not been accessed recently. It does so by throwing away data that can easily be read again (e.g. parts of executables), and by saving data that is unique (e.g. edited text) to a so-called paging server. This process is called swapping.

Data is swapped from workstation to paging server, or from paging server back to workstation. In the first case the workstation sends the data to the server. The server processes the data and generates an acknowledgement. In the second case the workstation sends a request to the server, triggering the generation of the data at the paging server.

Because of the correlation between successive pages being swapped to the server, this process is modelled to have hyper-exponential inter-arrival times. The same holds for requesting pages from the paging server.

4. In ongoing work, variable bit rate voice and video sources are used instead of constant bit rate sources. Their data is sent over the network in packets instead of using isochronous channels. 


\subsection{Character traffic}

With character traffic we mean applications like remote logging in (login, telnet), acknowledgements of Email reception, and the transmission of files between systems ( $\mathrm{ftp}$ and $\mathrm{TCp}$ ). The reason for taking them together is that the traffic they produce can be modelled in the same way and with the same parameters. The distribution used for the packet length is bimodal, the distribution for the inter-arrival time is exponential. This is realistic nowadays [15], and there is no reason to assume that this may be totally different in future.

Character traffic is exchanged between workstations, and between workstations and the gateway node.

\section{Simulation results}

In this section first the network configurations that have been simulated will be described. Next the types of curves that have been derived are introduced. At last the simulation results will be presented and discussed.

Table 1 describes the 3 different configurations that have been simulated.

\begin{tabular}{|c|c|c|}
\hline configuration & medium speed & network latency \\
\hline standard & $100 \mathrm{Mbit} / \mathrm{s}$ & $250 \mathrm{us}$ \\
long network & $100 \mathrm{Mbit/s}$ & $1 \mathrm{~ms}$ \\
high bandwidth & $1 \mathrm{Gbit} / \mathrm{s}$ & $250 \mathrm{us}$ \\
\hline
\end{tabular}

Table 1: the configurations used in the simulation

Except for a configuration representative for nowadays LANs/MANs, also a longer network and one with a higher bandwidth are investigated. These additional configurations are chosen because it is to be expected that networks will use higher medium speeds and span geographically larger areas in future.

In case of the dual-bus network DQDB, each bus has a medium speed of $100 \mathrm{Mbit} / \mathrm{s}$ resp. $1 \mathrm{Gbit} / \mathrm{s}$. The number of stations on each network is 25 .

The applications the workload model consists of can be divided into 2 groups, based on the way their data is transmitted:

- isochronous applications (voice and video telephony);

- asynchronous applications (file access, paging, character traffic).

At the moment the majority of the data generated in a campus environment is isochronous. However, the asynchronous applications are said to be the ones that will need more bandwidth in future. It is therefore not clear whether the isochronous or the asynchronous applications will be dominant in future. Because of this uncertainty 2 simulations have been performed for each configuration: one with $25 \%$ of the medium bandwidth used by isochronous applications (leaving $75 \%$ to asynchronous applications), and one with $75 \%$ of the bandwidth used by isochronous applications (leaving $25 \%$ to asynchronous applications).

It will now be explained why it is not interesting to derive performance measures for the is $\propto$ chronous traffic. Next the types of curves derived for the asynchronous traffic are discussed.

Isochronous traffic is handled in the same way in all three networks. Further, its performance does not depend on the traffic load. Because of these reasons it is not useful to investigate the performance of the isochronous traffic. We therefore limit ourselves to investigating the performance of the asynchronous traffic. The isochronous traffic can therefore be seen as a kind of 'background traffic' in our simulations.

Concerning the asynchronous traffic, two different types of curves have been derived:

- load/delay-curves: the mean asynchronous sojourn time as a function of the asynchronous load, given a certain quantity of isochronous traffic on the network;

- maximum-throughput curves: the maximum amount of asynchronous traffic that can be transmitted as a function of the quantity of isochronous traffic on the network. With 'maximum' we do not mean the absolute maximum amount of traffic the network can handle, but the maximum amount for which a certain Quality of Service (QoS) is still provided to the asynchronous traffic. The QoS we require: the mean sojourn time of the asynchronous traffic should not exceed $10 \mathrm{~ms}$.

The simulation results will now be discussed in detail.

\subsection{The standard configuration}

Figure 5 shows the asynchronous sojourn time as a function of the asynchronous load on the ring. Part of the error bars has not been drawn in order to improve the clearness of the picture. As can be seen in Figure 5, in case of $75 \mathrm{Mbit} / \mathrm{s}$ isochronous load, the performance of all networks is quite close. In case of $25 \mathrm{Mbit} / \mathrm{s}$, UCN performs slightly better than FDDI, while DQDB performs significantly better than both other networks. Figure 6 shows the maximum asynchronous throughput of the networks as a function of the isochronous load. This figure shows that DQDB allows a larger asynchronous throughput than FDDI and UCN in case the isochronous load is less than $75 \mathrm{Mbit} / \mathrm{s}$, while it allows less asynchronous throughput than UCN and FDDI in case of more isochronous traffic.

Because DQDB uses two busses of $100 \mathrm{Mbit} / \mathrm{s}$ each (while FDDI and UCN only use a single ring of this bit rate), one 

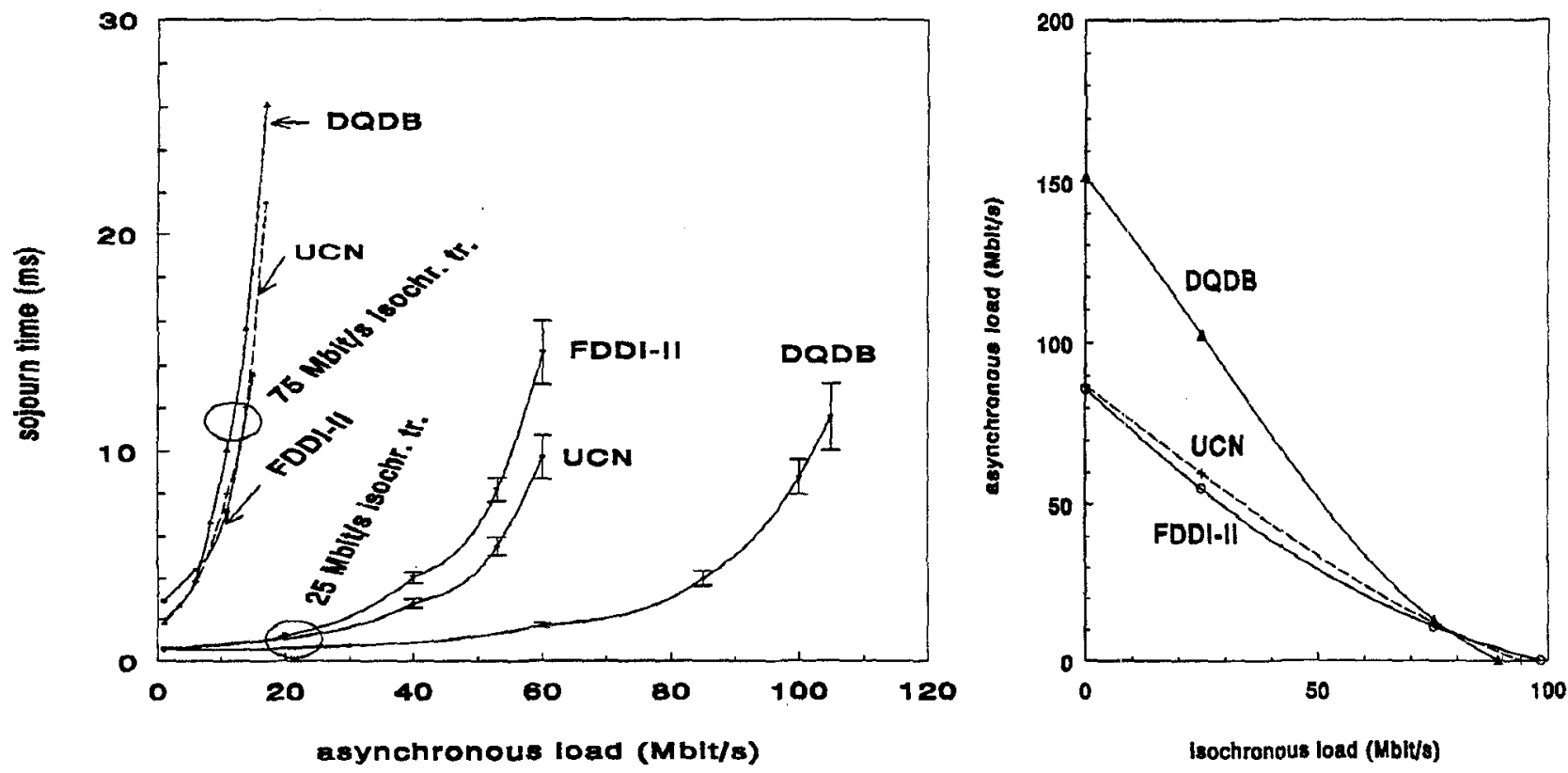

Figure 5: throughput/delay curves of the standard configuration

Figure 6: maximum throughput curves of the standard configuration

would expect DQDB to perform significantly better than UCN and FDDI. As can be seen, this is only the case for situations with little isochronous traffic. And even there, the performance is not as good as one might expect. In Figure 6 , e.g., one can see that in case of no isochronous traffic on the network, the maximum throughput of DQDB is $153 \mathrm{Mbit} / \mathrm{s}$, while this is $87 \mathrm{Mbit} / \mathrm{s}$ for UCN. This is 1.75 times as much, while one might have expected around twice the throughput. This effect can be explained as follows. First of all, the overhead is much larger in DQDB than in FDDI or UCN5. Secondly, since an odd number of workstation nodes is used, both busses in DQDB transfer a different amount of asynchronous load. This results in a different sojourn time for both busses. The sojourn time of the bus with the highest load (and therefore the highest sojourn times) will contribute most to the mean sojourn time, resulting in a larger mean value.

\subsection{The long network configuration}

In the second series of simulations, the networks used are four times larger than in the first simulation series. Figures 7 and 8 show the results of these simulations. Compared with the first simulation series, it can be concluded that all networks are scalable to larger network sizes. Further, the performance of FDDI slightly degrades compared to the shorter ring. This was to be expected because of two reasons: a larger propagation delay means that messages need more time to propagate, leading to a larger mean sojourn time. Further, the efficiency of the token ring decreases with increasing propagation delay.

The performance increase of UCN can be explained as follows. A larger propagation delay means that the basic slot size has to be increased, resulting in a reduced overhead for UCN. This performance advantage apparently exceeds the performance disadvantage of the increased propagation delay and the slightly increased fragmentation overhead.

Compared to the standard configuration, the performance of DQDB decreases when the network length is increased. This is caused by the increased propagation delay of the messages.

\subsection{High bandwidth configuration}

Figure 9 shows the throughput-delay curves for the $1 \mathrm{Gbit} / \mathrm{s}-$ configuration. The isochronous loads are $750 \mathrm{Mbit} / \mathrm{s}$ resp. $250 \mathrm{Mbit} / \mathrm{s}$. Figure 10 shows the maximum-throughput curves for this configuration. 

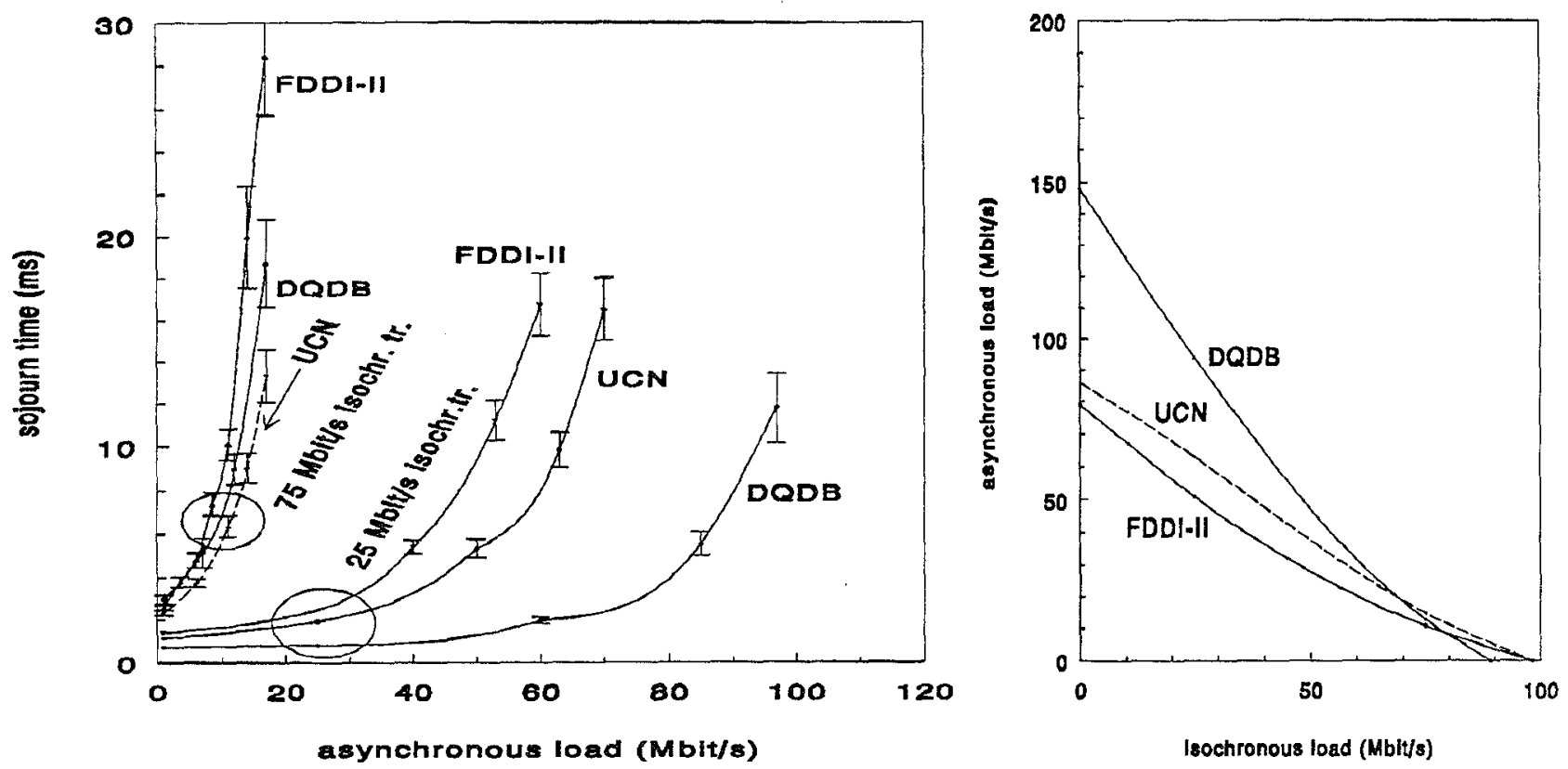

Figure 7: throughput/delay curves of the long network configuration

Figure 8: maximum throughput curves of the long network configuration

The first conclusion is that all networks are scalable with respect to the medium bandwidth. Further, compared to the standard configuration, all networks perform better at higher speed. One reason is as follows. The larger the bandwidth of the network is, the larger the applied load is, the larger consequently the number of sources is, and the better the sources statistically multiplex. Further, since the individual sources remain the same while the medium bandwidth has been increased, arriving bursts are transmitted faster (i.e. have a smaller transmission delay), resulting in a lower mean sojourn time.

\subsection{The maximum-throughput curves}

The maximum-throughput curves shown in Figures 6 and 8 are not straight lines, but they have slopes with decreasing derivatives. This curve form can be explained as follows.

In the ideal case the maximum-throughput curve is a straight line that runs from $100 \mathrm{Mbit} / \mathrm{s}$ isochronous load (in case of no asynchronous load) to $100 \mathrm{Mbit} / \mathrm{s}$ asynchronous load (in case of no isochronous load) ${ }^{6}$. For this line it holds that the total amount of traffic on the network equals the medium bit rate; traffic quantities above this line are not possible since

6. In case of DQDB the line runs to $200 \mathrm{Mbit} / \mathrm{s}$ asynchronous load; in case of $1 \mathrm{Gbit} / \mathrm{s}$ the values are 10 times as large. this would exceed the medium capacity. The actual curves in the figures therefore always have to be below this line.

The actual curves are the sum of the amount of isochronous traffic that can be transferred, and the amount of asynchronous traffic that can be transferred. For the isochronous traffic it holds that the amount equals the bandwidth that has been reserved for this type of traffic 7 . The efficiency of the isochronous traffic therefore does not depend on the amount of isochronous traffic. For asynchronous traffic, however, this does not hold: the more bandwidth available for asynchronous traffic, the better the statistical multiplexing of the asynchronous traffic, and the higher the efficiency is. Therefore, an increase of the asynchronous bandwidth of e.g. 10\% allows an increase of the asynchronous traffic of more than $10 \%$, because statistical multiplexing improves. As a result, the curves of Figure 6 and 8 have slopes with decreasing derivatives.

There is of course a limit on improving the statistical multiplexing of asynchronous traffic. This can be seen in Figure 10 (the $1 \mathrm{Gbit} / \mathrm{s}$ case): the curves are straight. Although the amount of asynchronous traffic is increased, the efficiency does not increase any further, resulting in a straight line. For the workload model used, statistical

7. Neglecting the fact that in FDDI and DQDB isochronous traffic is reserved in bunches of $96 \mathrm{resp} .48$ isochronous channels of $64 \mathrm{kbit} / \mathrm{s}$ each. 

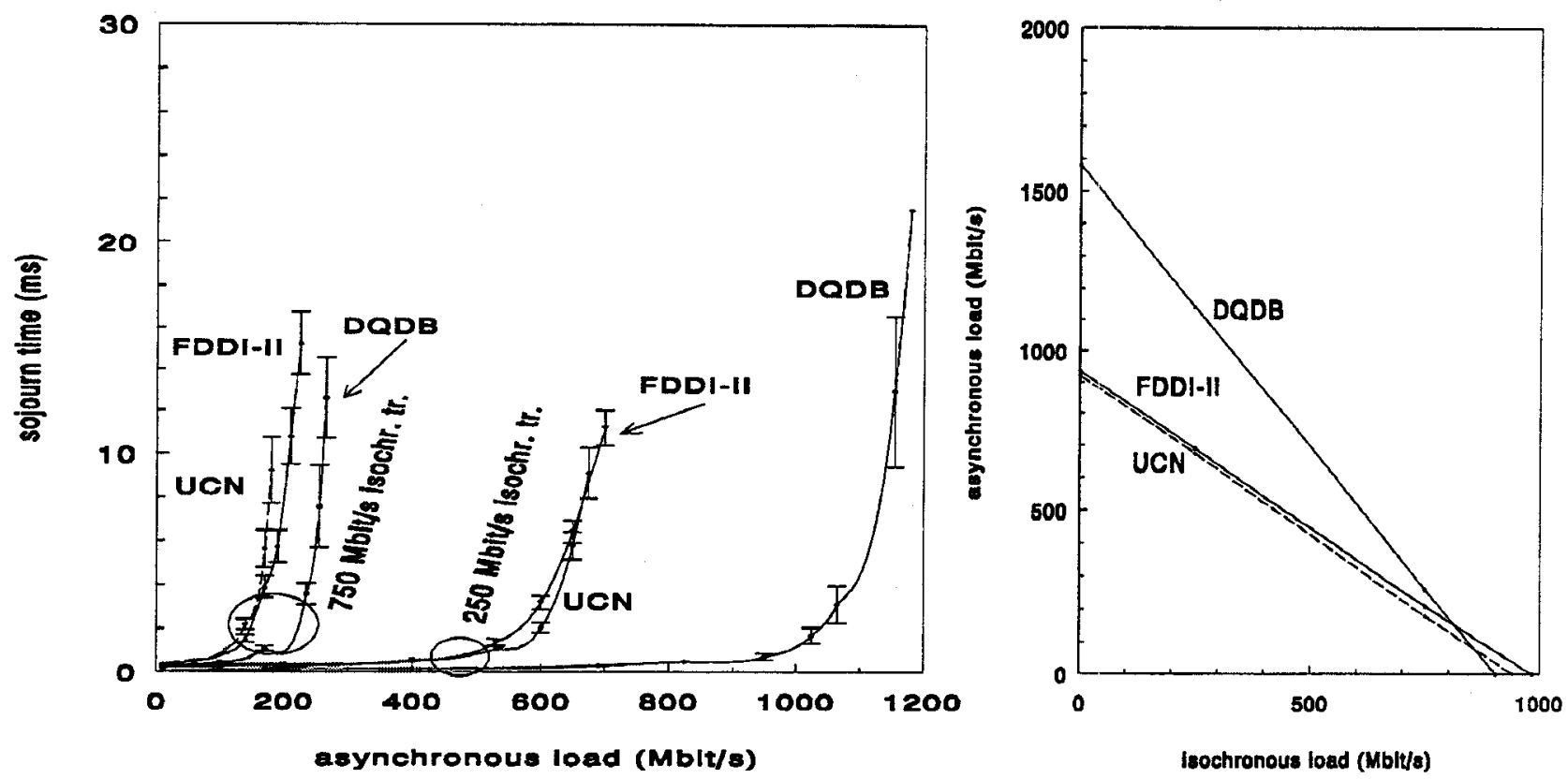

Figure 9: throughput/delay curves of the high bandwidth configuration
Figure 10: maximum throughput curves of the high bandwidth configuration multiplexing apparently reaches saturation for an asynchronous traffic quantity of around $100 \mathrm{Mbit} / \mathrm{s}$.

\section{Conclusions}

The token ring and the slotted ring are to a certain extent complementary with respect to the influence of the message length on the performance: the token ring outperforms the slotted ring for long variable-length messages, while the slotted ring outperforms the token ring for messages that exactly fit into a single slot. In this paper we argued that the advantages of both networks can be combined in a slotted ring in which the slot size can be adapted to the characteristics of the load. We presented the basic concepts of a network with this property, the Universal Channel Network (UCN), and we compared UCN with FDDI-II and DQDB.

The comparison has been done for three different configurations: a configuration representative for nowadays LANs/MANs, one with a substantially higher medium bandwidth, and one with an extended network size. As the workload model we used a detailed representation of what is seen on networks nowadays, extrapolated into the future.

In the simulations we performed, the 2 busses of DQDB each have the same capacity as the (single) ring of FDDI-II or UCN. Because of this, DQDB outperforms both FDDI-II and UCN. However, the advantage of DQDB is not as large as one would expect, and in case of much isochronous traffic there is no advantage at all. This is caused by the large overhead of DQDB, and by the fact that both busses in DQDB have a slightly different load quantity. Further, in most of the simulations performed, UCN shows a better performance than FDDI-JI. The simulations also showed that all three networks are scalable with respect to network length and medium bit rate.

\section{Acknowledgement}

Many thanks to $\mathrm{X}$. Hou for his part in designing the workload model, and to $M$. van der Zee for making the DQDB network model. We would also like to thank all our colleagues who allowed us to use their computers for running our simulations.

\section{References}

[1] Lambarelli L., Luvison L., Roffinella D., Sposini M., 'Service Integration in Wideband Local Area Networks: Problems and System Solutions', Proceedings Intnl. Tirrema Workshop on Digital Communications, Tirrema, 1985, North Holland, 1985. 
[2] Newmann S., The Communications Highway of the Future', IEEE Communications Magazine, October 1988, pp.45-50.

[3] Zafirovic-Vukotic M., Niemegeers I.G., Valk D.S., 'Performance analysis of slotted ring protocols in HSLAN's', IEEE Journal on Selected Areas in Communications, Vol. 6, No. 6, July 1988, pp. 10111024.

[4] Kamal A.E., Hamacher V.C., Utilizing bandwidth sharing in the slotted ring', IEEE Transactions on Computers, Vol. 39, No. 3, March 1990, pp. 289-299.

[5] Bux W., 'Local-area subnetworks: a performance comparison', IEEE Transactions on Communications, Vol. 29, No. 10, October 1981, pp. 1465-1473.

[6] Temple S., 'The Design of the Cambridge Ring', in 'Ring Technology Local Area Networks', Dallas I.N. and Spratt E.B. editors, Elsevier Science Publishers, 1984, pp.74-88.

[7] Zafirovic-Vukotic M., Performance modelling and evaluation of high-speed serial interconnection structures', Ph.D. thesis, University of Twente, Department of Computer Science, December 1988.

[8] Pasch H.L., Niemegeers I.G., 'A High-Speed Slotted Ring Access Mechanism with Dynamically Adaptive Slot Sizes', Memoranda Informatica 90-36, June 1990, University of Twente, Enschede, Netherlands.

[9] Greaves D., Hopper A., 'The Cambridge Backbone Network', Proc. EFOC/LAN '88, Amsterdam, June 1988, pp. 399-402.

[10] Conway R.W., Maxwell W.L., Miller L.W., "Theory of Scheduling', Addison-Wesley Publishing Company, 1967.

[11] Morris R.J.T., Wang Y.T., 'Some Results for Multiqueue Systems with Multiple Cyclic Servers', in Performance of Computer Communication Systems', Rudin H. and Bux W. editors, Elsevier Science Publishers, 1984, pp.245-258.

[12] Pasch H.L., Niemegeers I.G., 'A High-Speed Slotted Ring Access Mechanism with Dynamically Adaptive Slot Sizes', EFOC/LAN 91, London, June 19-21, 1991, LAN proceedings, pp 42-47 \& pp 248-253.

[13] Pasch H.L., Niemegeers I.G., 'A Simulation Study on a High-Speed Slotted-Ring Access Mechanism with Dynamically Adaptive Slot Sizes, Local Computer Networks 1991, October 14 - 17, 1991, Minneapolis, USA, pp. 106-113.

[14] Abeysundara B.W., Kamal A.E., 'High-Speed Local Area Networks and Their Performance: A Survey', ACM Computing Surveys, Vol. 23, No.2, June 1991, pp. 221-264.

[15] Gusella R., 'Characterizing the variability of arrival processes with indexes of dispersion', IEEE Journal on Selected Areas in Communications, Vol. 9, No.2, February 1991, pp. 203-211.

[16] Jain R., Routhier S.A., 'Packet trains - Measurements and a New Model for Computer Network Traffic',
IEEE Joumal on Selected Areas in Communications, Vol. 4, No. 6, September. 1986, pp. 986-995.

[17] Shoch J.F., Hupp J.A., 'Measured Performance of an Ethernet local area network', Communications of the ACM, Vol. 23, No. 12, December. 1980, pp. 711-721.

[18] Lazowska E.D., Zahorjan J., File access performance of diskless workstations'. ACM Transactions on Computer Systems, Vol. 4, No. 3, August. 1986, pp. 238-271.

[19] Vyncke E., Danthine A., 'A realistic simulation of a wideband backbone network', Kuehn P. (ed.), New communication services: a challenge to computer technology, Elsevier Science Publishers, 1986, pp. 176-181.

[20] Pasch H.L., Niemegeers I.G., 'A performance analysis of a high-speed slotted-ring access mechanism with dynamically adaptive slot sizes', Globecom '91, Phoenix, Arizona, USA, December 2-5, 1991, pp. 1102-1109.

[21] Pasch H.L., Niemegeers I.G., 'A workload model of a future integrated services campus network', to appear as Memorandum Informatica, University of Twente, Enschede, Netherlands.

[22] OSI international standard / ANSI standard X3T9, Fibre Distributed Data Interface (FDDI), Part 2: token ring media access control (MAC)', 1989.

[23] Draft proposed American national standard X3T9, 'FDDI hybrid ring control', August 1989.

[24] IEEE proposed standard 802.6, 'Distributed Queue Dual Bus (DQDB) subnetwork of a metropolitan area network (MAN)', July 1990. 